\title{
Feedforward Inhibitory Control of Sensory Information in Higher-Order Thalamic Nuclei
}

\author{
Philippe Lavallée, ${ }^{1}$ Nadia Urbain, ${ }^{1}$ Caroline Dufresne, ${ }^{1}$ Hajnalka Bokor, ${ }^{2}$ László Acsády, ${ }^{2}$ and Martin Deschênes ${ }^{1}$ \\ ${ }^{1}$ Centre de Recherche Université Laval Robert-Giffard, Hôpital Robert-Giffard, Québec, Canada G1J 2G3, and 2Institute of Experimental Medicine, \\ Hungarian Academy of Sciences, Budapest H-1450, Hungary
}

\begin{abstract}
Sensory stimuli evoke strong responses in thalamic relay cells, which ensure a faithful relay of information to the neocortex. However, relay cells of the posterior thalamic nuclear group in rodents, despite receiving significant trigeminal input, respond poorly to vibrissa deflection. Here we show that sensory transmission in this nucleus is impeded by fast feedforward inhibition mediated by GABAergic neurons of the zona incerta. Intracellular recordings of posterior group neurons revealed that the first synaptic event after whisker deflection is a prominent inhibition. Whisker-evoked EPSPs with fast rise time and longer onset latency are unveiled only after lesioning the zona incerta. Excitation survives barrel cortex lesion, demonstrating its peripheral origin. Electron microscopic data confirm that trigeminal axons make large synaptic terminals on the proximal dendrites of posterior group cells and on the somata of incertal neurons. Thus, the connectivity of the system allows an unusual situation in which inhibition precedes ascending excitation resulting in efficient shunting of the responses. The dominance of inhibition over excitation strongly suggests that the paralemniscal pathway is not designed to relay inputs triggered by passive whisker deflection. Instead, we propose that this pathway operates through disinhibition, and that the posterior group forwards to the cerebral cortex sensory information that is contingent on motor instructions.
\end{abstract}

Key words: barrel; inhibition; thalamus; trigeminal; vibrissa; whisker; zona incerta

\section{Introduction}

The faithful relay of peripheral information in sensory-specific thalamic nuclei (also called first-order nuclei) is ensured by large excitatory terminals with multiple synaptic contacts on the proximal dendrites of relay cells. In association (or higher order) thalamic nuclei, however, responses of relay cells to sensory stimuli are usually delayed and more labile and are suppressed by silencing the cerebral cortex (Bender, 1983; Diamond et al., 1992b). This is the case, for instance, of the relay cells in the posterior thalamic nuclear group $(\mathrm{Po})$ in rodents that, despite receiving a significant input from the interpolaris nucleus of the trigeminal complex (SpVi), respond poorly to whisker deflection (Chiaia et al., 1991; Diamond et al., 1992a; Sosnik et al., 2001). However, when present, responses occur at long latencies ( $\sim 20 \mathrm{~ms})$ and are of much lower magnitude than those observed in the first-order somatosensory nucleus, the ventral posterior medial nucleus (VPM). Because whisker-evoked responses in Po are suppressed during barrel cortex inactivation, it was suggested that sensory transmission could depend on cortical feedback (Diamond et al., 1992b).

Anatomical studies have shown that Po receives the majority

Received April 28, 2005; revised July 4, 2005; accepted July 6, 2005.

This work was supported by the Canadian Institutes of Health Research, the Hungarian Scientific Research Fund (OTKA T 049100), and the Wellcome Trust. We thank Krisztina Faddi and Gyözö Goda for their excellent technical assistance, and Dr. K. G Bainbridge for the rabbit anti-calbindin and rabbit anti-parvalbumin antisera. We also thank Dr. Paul De Koninck for helpful comments on a previous version of this manuscript.

Correspondence should be addressed to Martin Deschênes, Centre de Recherche, Université Laval Robert-Giffard, 2601 de la Canardière, Québec, Canada G1J 2G3. E-mail: martin.deschenes@crulrg.ulaval.ca.

D01:10.1523/JNEUROSCI.2301-05.2005

Copyright $\odot 2005$ Society for Neuroscience $\quad$ 0270-6474/05/257489-10\$15.00/0 of its ascending input from the axonal branches of large-sized, multiwhisker-responsive cells located in the SpVi (Jacquin et al., 1989; Williams et al., 1994; Veinante and Deschênes, 1999; Veinante et al., 2000a). In addition, Po receives sparse afferents from the principal trigeminal nucleus $(\mathrm{PrV})$ that are mostly concentrated in the caudal part of the nucleus (for connections involved in vibrissal information processing in Po, see Fig. 1). However, no study has documented the ultrastructure of trigeminothalamic synapses in Po. The posterior group also receives large excitatory terminals from layer 5 of the barrel field (Hoogland et al., 1991; Veinante et al., 2000), which were suggested to play a role in the dependence of sensory-evoked responses on the integrity of the neocortex in Po (Diamond, 1995). On their way to the thalamus, $\mathrm{PrV}$ and $\mathrm{SpVi}$ axons also terminate profusely in the zona incerta (ZI), a ventral thalamic region principally composed of GABAergic neurons (Lin et al., 1990; Kim et al., 1992; Kolmac and Mitrofanis, 1998). In turn, incertal cells project to Po, in which they make large GABAergic synaptic contacts on the proximal dendrites of relay cells (Barthó et al., 2002). Thus, the connectivity of prethalamic afferents in Po provides a substrate for feedforward inhibition that could depress responses to sensory stimuli. Alternatively, ZI neurons might discharge spontaneously at high rates, which could explain why whisker-evoked EPSPs in Po rarely reach spike threshold. Whatever the case, both mechanisms imply that ZI lesion should enhance whisker-evoked responses in Po. This is indeed the result reported in a recent study in ZIlesioned rats (Trageser and Keller, 2004). However, the mechanisms by which ZI blocks sensory responses and whether this inhibitory effect still persists after cortical inactivation remain open questions. In the present study, we used extracellular and 
intracellular recordings and electron microscopy to examine how ZI and barrel cortex control sensory transmission in Po.

\section{Materials and Methods}

Animal preparation and recordings. Experiments were performed in male rats (Sprague Dawley, 250-300 g) in accordance with federally prescribed animal care and use guidelines. The University Committee for Animal Use in Research approved all experimental protocols. Rats were anesthetized with ketamine $(75 \mathrm{mg} / \mathrm{kg}$ ) plus xylazine $(5 \mathrm{mg} / \mathrm{kg})$, the left facial nerve was cut, and the animal was placed in a stereotaxic apparatus. In different experiments, openings were made over the thalamus and barrel cortex for the descent of recording and stimulating electrodes. Once surgery was completed, the nape of the neck and incised tissue were infiltrated with a long-lasting local anesthetic (Marcaine 1\%) to reduce animal discomfort. During the recording sessions, animals frequently displayed spontaneous twitches of the right whiskers, briskly reacted to a moderate pinch of the hindlimb, but otherwise remained motionless, indicating that they did not experience any discomfort. An additional dose of anesthetics (ketamine, $20 \mathrm{mg} / \mathrm{kg}$ ) was given when smallamplitude whisking movements were noticed. In other rats, recordings were performed under urethane anesthesia $(1.4 \mathrm{~g} / \mathrm{kg})$ according to the above described protocol.

Single units were recorded in ZI and Po with glass micropipettes $(0.5-1 \mu \mathrm{m})$ filled with potassium acetate $(0.5 \mathrm{M})$ and a biotinylated tracer (Neurobiotin or biotin dextran amine) (2\% BDA, 10,000 molecular weight; Molecular Probes, Eugene, OR). Micropipettes were lowered vertically in ZI but at a lateral angle of $30^{\circ}$ from the vertical in Po. Cell location in ZI was assessed by the juxtacellular labeling of single units (Pinault, 1996) or by making small extracellular deposits of BDA (200 ms current pulses, $50 \mathrm{nA}, 5 \mathrm{~min}$ ). Posterior group neurons were labeled either juxtacellularly or intracellularly. Incertal cells that project to Po were identified by antidromic invasion using the collision test. At the end of the experiments, rats were perfused under deep anesthesia with saline, followed by a fixative containing $4 \%$ paraformaldehyde and $0.5 \%$ glutaraldehyde in phosphate buffer $(\mathrm{PB})(0.1 \mathrm{M}), \mathrm{pH}$ 7.4. Brains were removed, cryoprotected in $30 \%$ sucrose, and cut coronally at $70 \mu \mathrm{m}$ on a freezing microtome. Sections were processed for cytochrome oxidase and Neurobiotin histochemistry according to standard protocols that were described in detail previously (Veinante et al., 2000a).

Whisker stimulation. Whiskers were cut at $2 \mathrm{~cm}$ from the skin, and air jets (50 ms, 40 psi) were generated by a Picospitzer (General Valve, Brookshire, TX) connected to a pipette (tip diameter, $\sim 500 \mu \mathrm{m}$ ). The pipette was positioned at a distance of $3 \mathrm{~cm}$ of the vibrissae, in front of the animal, and the blast was directed away from the rat's face to avoid as much as possible stimulating other orofacial afferents. The delay between the command voltage and the actual motion of vibrissae was measured by placing a piezoelectric film (Measurement Specialties, Fairfield, NJ) at the same distance from the tip of the pipette. This delay $(9 \mathrm{~ms})$ was subtracted from the recordings to build poststimulus time histograms (PSTHs) of sensory-evoked responses.

Kainic acid lesion. Kainic acid was injected iontophoretically to restrict the extent of ZI lesions in the ventral thalamus. First, the whiskerresponsive region of $\mathrm{ZI}$ was located by single-unit recordings. Then, a micropipette (tip diameter, $30 \mu \mathrm{m}$ ) filled with kainic acid (1\%) in Tris buffer, $\mathrm{pH}$ 8.3, was lowered in that same region, and the toxin was ejected with negative current pulses of $1 \mu \mathrm{A}$ ( $2 \mathrm{~s}$ duty cycle) for $20 \mathrm{~min}$. After the injection, the skin was sutured, rats were given analgesics (Anafen; 5 $\mathrm{mg} / \mathrm{kg}$ ), and they were returned to the animal facilities. Electrophysiological recordings were performed 2-3 d later according to the procedure described above. At the end of the experiments, rats were perfused, and alternate sections were processed for Nissl staining and neuronal-specific nuclear protein $(\mathrm{NeuN})$ immunohistochemistry. After preincubation for $1 \mathrm{~h}$ in PBS with 3\% normal goat serum and $0.3 \%$ Triton X-100, sections were incubated overnight in the same mixture containing NeuN antibody (1:200 dilution; mouse monoclonal IgG; Chemicon, Temecula, CA). A secondary antibody conjugated to Alexa 488 was used to reveal immunostaining.

Cortical lesion. The barrel cortex was lesioned by the application of a small crystal of silver nitrate over the pia. The crystal was left in place for
10 min to allow diffusion of the chemical to the deep layers. Then, the cortical surface was abundantly rinsed with saline. Silver nitrate is a strong cauterizing agent that burns tissue; this type of lesion does not produce any bleeding or tissue swelling. In control experiments $(n=2)$, in which electrocorticograms were recorded, silver nitrate produced an almost immediate flattening of the electrographic trace (supplemental Fig. S1, available at www.jneurosci.org as supplemental material). The rapid onset of cortical silencing likely relies on oxygen depletion produced by the cauterization of arterial blood vessels. After aldehyde fixation, the burnt tissue becomes brownish but turns black after cytochrome oxidase processing. The extent of the lesion clearly showed up in the histologic material as a deep black region.

Data analysis. Responses to whisker deflection were collected in PSTHs with bin width of $1 \mathrm{~ms}$. When PSTHs included 20 responses, they were expressed in counts per bin; when they included a variable number of sweeps (20-50 responses), they were scaled in probabilities. The presence of statistically significant responses was assessed by comparing firing rates within a $40 \mathrm{~ms}$ time window after stimulus onset with prestimulus firing rates estimated over a $100 \mathrm{~ms}$ period ( $\alpha=0.05$; one-tailed $t$ test). The latency of whisker-evoked responses was estimated as the bin corresponding to 0.5 of peak value of the PSTH (minus background) after three bin smoothing with a boxcar kernel (for the rationale of this method, see Discussion by Sosnik et al., 2001). Data analysis was performed with the Neuroexplorer (Plexon, Dallas, TX) and Excel (Microsoft, Redmond, WA) software. Unless otherwise stated, results are reported as mean $\pm \mathrm{SD}$.

Retrograde labeling. FluoroGold was injected iontophoretically in the Po of three rats. The tracer was delivered by passing depolarizing current pulses ( $300 \mathrm{nA}, 2 \mathrm{~s}$ duty cycle for $20 \mathrm{~min}$ ) through micropipettes (diameter, $15 \mu \mathrm{m}$ ) filled with $2 \%$ FluoroGold dissolved in cacodylate buffer $(0.1 \mathrm{M}), \mathrm{pH}$ 7. After a survival period of $2 \mathrm{~d}$, rats were perfused as above, and sections were stained for cytochrome oxidase and processed for FluoroGold immunohistochemistry. After preincubation for $1 \mathrm{~h}$ in PBS with $3 \%$ normal goat serum and $0.3 \%$ Triton X-100, sections were incubated overnight in the same mixture containing an anti-FluoroGold antiserum (1:8000; Chemicon). The antibody was revealed using a peroxidase-labeled secondary antibody (goat IgG; Chemicon) and nickel-3,3'-diaminobenzidine tetrahydrochloride (Ni-DAB) as a substrate.

Anterograde tracing and electron microscopy. Three rats were anesthetized with Equithesin (chlornembutal, $0.3 \mathrm{ml} / 100 \mathrm{~g}$ ), and the whiskerresponsive region of $\mathrm{SpVi}$ was identified by unit recordings. Then, a micropipette (tip diameter, $\sim 20 \mu \mathrm{m}$ ) containing BDA (10\% in saline) was lowered in that same region, and the tracer was ejected with positive current pulses ( $2 \mathrm{~s}$ duty cycle) of $0.5-4.0 \mu \mathrm{A}$ for $30 \mathrm{~min}$. After a survival period of 5-7 d, rats were deeply anesthetized with Equithesin and perfused with physiological saline $(2 \mathrm{~min})$, followed by $100 \mathrm{ml}$ of fixative containing $2 \%$ paraformaldehyde plus $0.5 \%$ glutaraldehyde in acetate buffer, pH 6.0 (3 min), and finally with $400 \mathrm{ml}$ of fixative containing $2 \%$ paraformaldehyde plus $0.5 \%$ glutaraldehyde in borate buffer, $\mathrm{pH} 8.5$ (50 $\mathrm{min})$. Coronal sections of the thalamus (50 $\mu \mathrm{m}$ thick) were cut on a vibratome, washed, cryoprotected in $30 \%$ sucrose in $0.1 \mathrm{M} \mathrm{PB}$ overnight, and freeze thawed in an aluminum-foil boat over liquid nitrogen. Injection sites were visualized on vibratome-cut horizontal sections of the brainstem after Nissl staining.

Sections through the thalamus were incubated with the avidin biotinylated-horseradish peroxidase complex (ABC; Vector Laboratories, Burlingame, CA) in Tris-buffered saline (TBS) for $2 \mathrm{~h}$ and developed in $\mathrm{Ni}-\mathrm{DAB}$. After visualization of the tracer with $\mathrm{Ni}-\mathrm{DAB}$, different sections were incubated with rabbit anti-calbindin or rabbit anti-parvalbumin antisera (1:2000) (Baimbridge and Miller, 1982) for $2 \mathrm{~d}$; then they were incubated with a goat anti-rabbit $\operatorname{IgG}(1: 200)$ for $2 \mathrm{~h}$, a rabbit peroxidaseanti peroxidase complex (1:600; Sternberger Monoclonals, Lutherville, $\mathrm{MD}$ ) overnight, and developed with DAB. A similar protocol was used to reveal GABA immunoreactivity in other sections (mouse anti-GABA antibody, 1:50) (Szabat et al., 1992). The specificity of all antisera has been extensively investigated in their laboratories of origin. All sections were osmicated $\left(1 \% \mathrm{OsO}_{4}\right.$ in $\mathrm{PB}$ for $30 \mathrm{~min}$, with $7 \%$ sucrose added to preserve color difference), dehydrated in ethanol and propylene oxide, 
and embedded in Durcupan (ACM; Fluka, Buchs, Switzerland). During dehydration, sections were treated with $1 \%$ uranyl acetate in $70 \%$ ethanol for $40 \mathrm{~min}$.

For fluorescence microscopy, sections were cut on a vibratome, incubated with rabbit anti-type 2 vesicular glutamate transporter (vGlut2) (1:3000; Chemicon) and Alexa 594-conjugated goat anti-rabbit IgG (1: 200; Molecular Probes). BDA was visualized with Alexa 488-conjugated streptavidin (1:3000; Molecular Probes).

To examine the ultrastructure of BDA-labeled terminals, sections were cut with a vibratome and incubated with $\mathrm{ABC}$, and the signal was amplified with a biotinyl tyramide reagent (1:50, 15 min; PerkinElmer Life Sciences, Boston, MA). Then, sections were incubated overnight in $1 \mathrm{~nm}$ gold-conjugated streptavidin (1:50; Aurion, Wageningen, The Netherlands) dissolved in TBS containing $0.8 \%$ BSA and $0.1 \%$ gelatin. Thereafter, sections were postfixed in $2 \%$ glutaraldehyde in TBS and silver intensified with Aurion R-Gent intensification kit. All sections were treated with $\mathrm{OsO}_{4}$ ( $1 \%$ for $45 \mathrm{~min}$ for $\mathrm{DAB}$ staining; $1 \% 1 \mathrm{~min}$; and $0.5 \%$ for 20 min at $4^{\circ} \mathrm{C}$ for immunogold staining in $0.1 \mathrm{M} \mathrm{PB}$ ), dehydrated as described above, and embedded in Durcupan.

Selected sections containing labeled terminals were reembedded, cut at $60 \mathrm{~nm}$ with an Ultramicrotome (Reichert), and mounted on copper or nickel grids. Postembedding GABA immunostaining was performed on nickel grids according to the protocol of Somogyi et al. (1985).

Light microscopy pictures were taken with a digital camera (DP 70; Olympus Optical, Tokyo, Japan). Electron micrographs were taken on a Hitachi (Tokyo, Japan) 7100 electron microscope, the negatives were scanned, and brightness and contrast were adjusted if necessary using Adobe Photoshop 7.0 (Adobe Systems, San Jose, CA). Measurements were made using the software AnalySIS.

\section{Results}

The posterior thalamic nuclear group in rats is a large nucleus that surrounds the medial and caudal aspects of the ventrobasal complex (Paxinos and Watson, 1998). It does not exhibit obvious cytoarchitectonic subdivisions, but it is generally believed that Po contains distinct functional regions (Diamond, 1995). That part of Po we shall be concerned with consists of a shell-like region at the margin of the VPM, in which neurons receive vibrissal input from the SpVi (Fig. 1) (Diamond et al., 1992a; Williams et al., 1994; Veinante et al., 2000a).

\section{Whisker-responsive ZI cells that project to Po discharge at high rates}

Under light ketamine anesthesia, we recorded the activity of 75 whisker-responsive cells in ZI, among which 33 were backfired from Po at a mean latency of $1.04 \pm 0.23 \mathrm{~ms}$. On the basis of single-cell labeling $(n=11)$ or electrode tract reconstruction after small deposits of BDA, $86 \%$ of these neurons (i.e., 65 cells) were located in the ventral division of $\mathrm{ZI}(\mathrm{ZIv})$. As the recording electrode traversed ZI, two distinct patterns of spontaneous activity were observed: in dorsal ZI (ZId), cells fired at low rates $(<8$ $\mathrm{Hz}$ ) single spikes and short trains of action potentials, whereas ZIv neurons displayed regular discharges of much higher frequency. Figure $2 A-E$ shows a representative unit that discharged spontaneously at $33 \mathrm{~Hz}(A, B)$ that was invaded antidromically from $\operatorname{Po}(C)$, responded to whisker deflection produced by an air jet $(D)$, and was located in $\operatorname{ZIv}(E)$. The histogram in $F$ shows the distribution of mean firing rates that were computed for 65 whisker-responsive ZIv units by compiling interspike intervals over 1 min periods. The mean firing rate among this population was $25.81 \pm 17.25 \mathrm{~Hz}$. For 10 of these units, which were also backfired from Po, we used air-jet stimulation to build the population PSTH shown in $G$. Responses were robust, involved multiple vibrissae, and had a mean onset latency of $4.57 \pm 0.65 \mathrm{~ms}$ (computed as the bin value corresponding to $50 \%$ of the first peak in individual PSTHs).

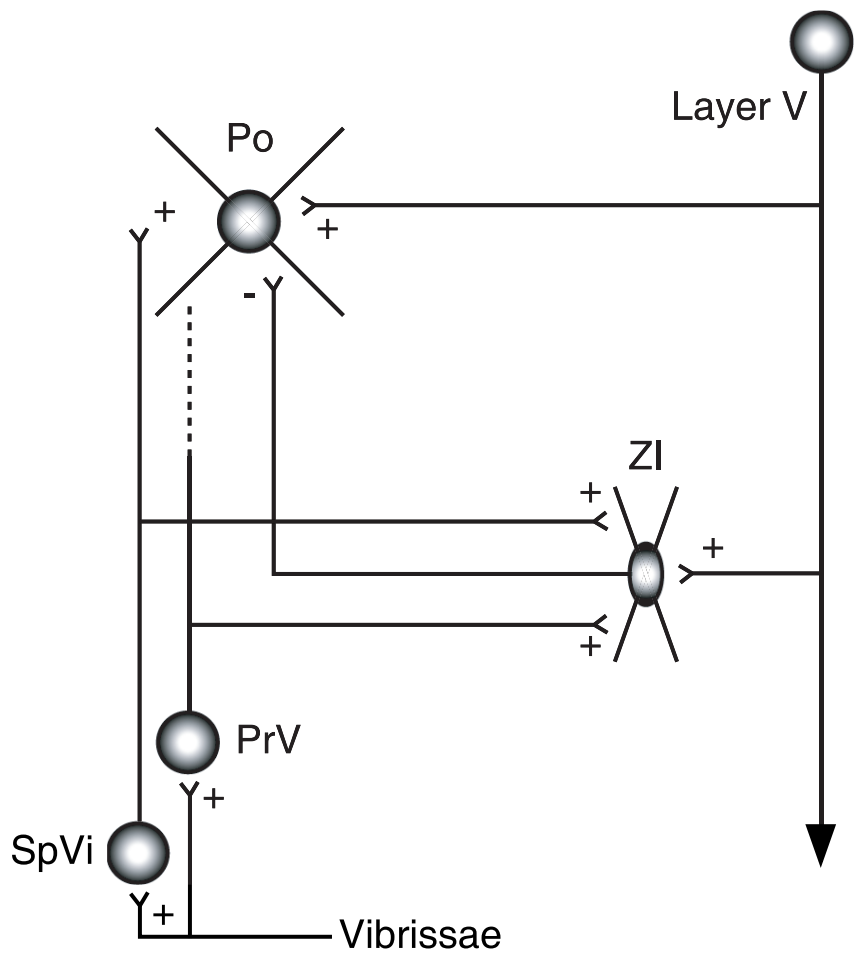

Figure 1. Diagram of connections involved in vibrissal information processing in $\mathrm{Zl}$ and $\mathrm{Po}$. These nuclei receive vibrissal input from the axonal branches of large-sized, multiwhiskerresponsive cells located in the PrV and the SpVi (Jacquin et al., 1989; Williams et al., 1994; Veinante and Deschênes, 1999; Veinante et al., 2000a). Both groups of axons terminate profusely in Zl, but their projections in Po differ; that provided by the PrV is sparse and principally concentrated in the caudal part of the nucleus, whereas that issued from the SpVi is dense and forms a shell-like terminal field at the margin of the VPM (Williams et al., 1994; Veinante et al., 2000a). Like most higher-order thalamic nuclei, Po receives a dual corticothalamic input: one that arises from lamina 6 cells in cortical areas innervated by Po axons (omitted for simplicity), and another one from layer 5 cells that are exclusively located in the granular and dysgranular zones of the barrel field (Hoogland et al., 1987; Bourassa et al., 1995; Veinante et al., 2000b). Latter projection consists of collaterals of corticofugal cells whose main axons also project to the striatum, tectum, Zl, pontine nuclei, and trigeminal nuclei. These collaterals do not supply branches in the reticular thalamic nucleus (Veinante et al., 2000b) but make large synaptic contacts on the proximal dendrites of Po neurons (Hoogland et al., 1991). Inhibitory inputs from the reticular thalamic nucleus and APT are not shown. The dashed line connecting PrV to Po indicates a sparse contribution to the dorsal part of $\mathrm{P}_{0}$, which contains the whisker representation.

Although histological material confirmed the location of the stimulating electrode in Po (Fig. $2 \mathrm{H}$ ), it remained possible that an unknown proportion of ZIv neurons were activated by current diffusion or the activation of passing fibers. To further verify the origin of incertal projections, FluoroGold was injected in the Po of three rats. Two injections were confined in Po, and, in both cases, the vast majority of retrogradely labeled cells were found in ZIv (Fig. $2 I, J$ ). Thus, this result supports the finding that incertal afferents to Po principally arise from ZIv.

Because most previous studies of Po neurons have been performed in urethane-anesthetized rats, we also examined how incertal cells discharge under that type of anesthesia. The recording of 42 incertal neurons in three rats did not reveal any clear difference in the rate of discharges between ZId and ZIv neurons. All cells discharged at low rates $(<5 \mathrm{~Hz})$ but still responded briskly to whisker deflection (supplemental Fig. S2, available at www. jneurosci.org as supplemental material). Responses occurred at short latency $(4.75 \pm 1.32 \mathrm{~ms})$ but were less sustained than in rats maintained under light ketamine anesthesia. 

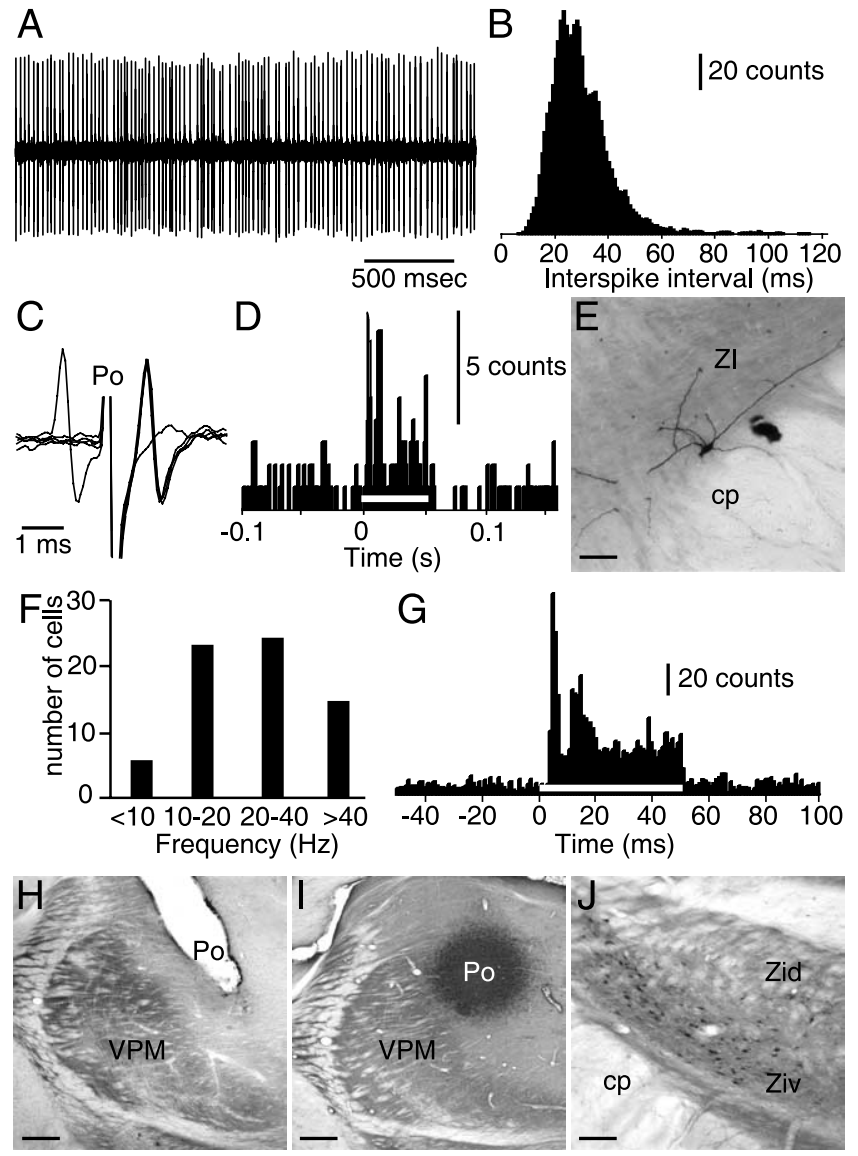

Figure 2. Electrophysiological and anatomical features of whisker-responsive ZI cells that project to $\mathrm{Po}_{0}$. The sustained discharges of a representative $\mathrm{Zl}$ unit is shown in $\boldsymbol{A}$, with the distribution of interspike intervals compiled during a 1 min period in $\boldsymbol{B}$ (mean firing rate, $33 \mathrm{~Hz}$ ). This unit was backfired from Po (arrow points to a collision in $C$ ), it responded to whisker deflection (PSTH in D; the white bar indicates the air-jet stimuli), and it was found in Zlv after juxtacellular labeling with Neurobiotin $(\boldsymbol{E})$. The distribution of mean firing rates among a population of 65 whisker-responsive Zlv cells is shown in $\boldsymbol{F}$ (mean firing rate, $25.81 \pm 17.25 \mathrm{~Hz}$ ). Population PSTH in $\mathbf{G}$ shows responses evoked by whisker deflection (50 ms air jet) in 10 Zlv units that were also backfired from $\mathrm{P}_{0}$. The location of the stimulating electrode is shown in $\boldsymbol{H}$ $I, J$, The ventral division of $Z I$ is the main source of projection to $\mathrm{Po}$ as confirmed by the injection of FluoroGold into the dorsal $\mathrm{P}_{0}(I)$. The vast majority of retrogradely labeled neurons are found in Zlv $(\boldsymbol{J})$. Scale bars: $\boldsymbol{E}, 50 \mu \mathrm{m} ; \boldsymbol{H}, \boldsymbol{I}, 200 \mu \mathrm{m} ; \boldsymbol{J}, 100 \mu \mathrm{m}$. cp, Cerebral peduncle.

\section{Kainic acid lesion of ZI disinhibits Po neurons}

Because ZI axons terminate on the proximal dendrites of Po neurons (Barthó et al., 2002), one would expect the latter neurons to be strongly depressed by the sustained discharges of incertal cells. In extracellular recordings, under light ketamine anesthesia, the rate of spontaneous discharges in Po was indeed very low $(<0.2$ $\mathrm{Hz}$ ) (Fig. 3A), and neurons did not respond at all or responded very inconsistently to somatic stimuli. Most attempts to get PSTHs with statistically significant $\mathrm{ON}$ responses to whisker deflection failed or barely reached 5\% significance level (one-tailed $t$ test). This contrasted with the short-latency, consistent $\mathrm{ON}$ responses evoked in VPM cells by the same stimuli (supplemental Fig. S3, available at www.jneurosci.org as supplemental material). Intracellular recordings $(n=22)$ revealed Po neurons with high membrane noise levels, approximately five times larger than those observed in the VPM (Fig. 3B). The average variance of voltage fluctuations measured at rest amounted to $150 \pm 43$ and $29 \pm 16 \mathrm{mV}^{2}$ in Po $(n=10)$ and VPM $(n=10)$ cells, respectively. When depolarizing current was injected in Po neurons, spike discharges rapidly inactivated, revealing a steady barrage of fast
A
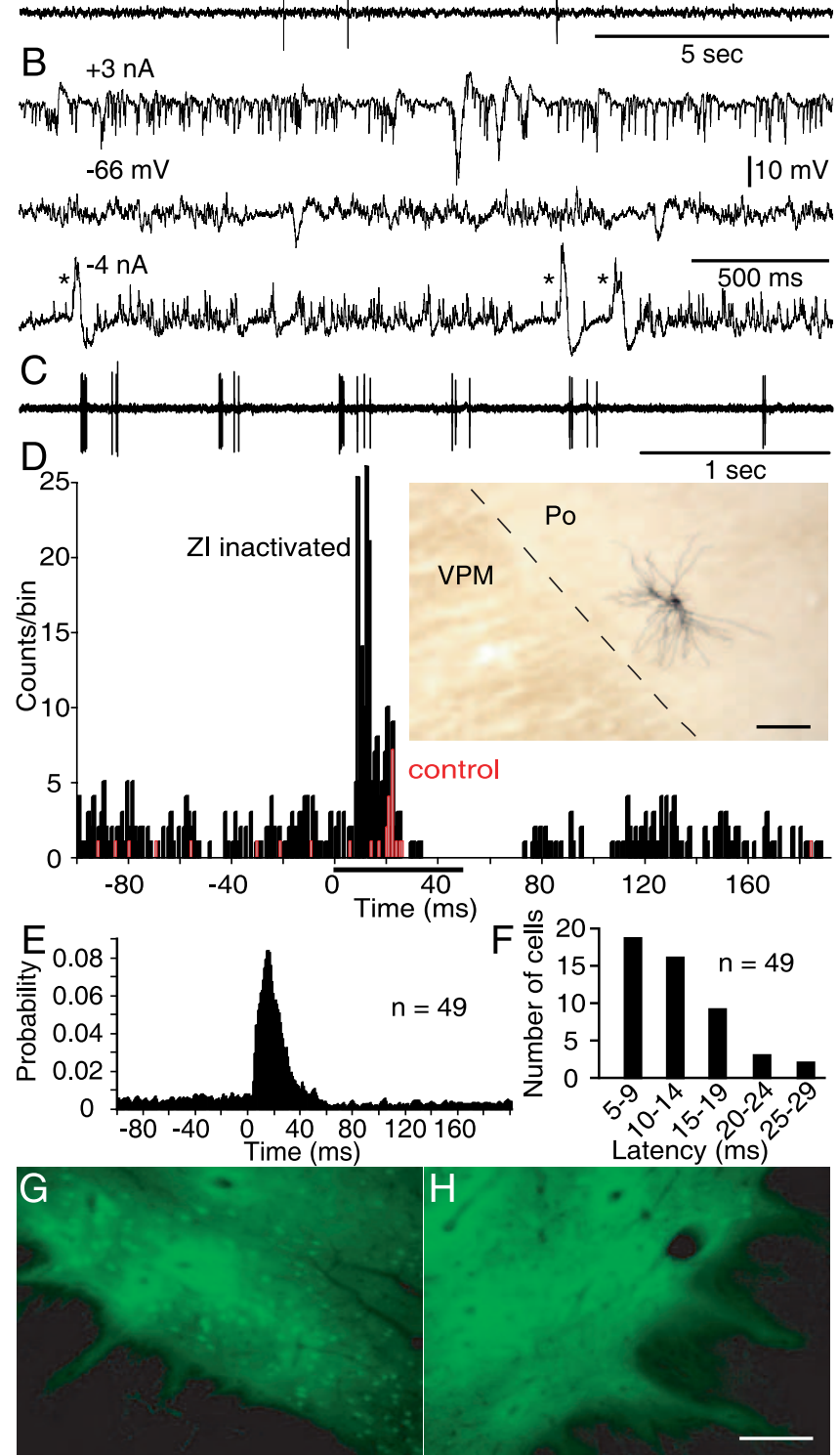
Latency (ms)

Figure 3. Kainic acid lesion of ZI disinhibits Po neurons. $\boldsymbol{A}$, In normal rats, Po cells discharge spontaneously at low rates. Intracellular recordings reveal that the membrane potential of $\mathrm{P}_{0}$ cells is continuously riddled with IPSPs (alternating current recordings in $\boldsymbol{B}$; bandwidth, $10 \mathrm{~Hz}$ to $3 \mathrm{kHz}$ ), which reverse polarity at $-66 \mathrm{mV}$. The large depolarizations during membrane hyperpolarization are low-threshold spikes (asterisks). C, In ZI-lesioned rats, Po cells discharge rhythmic trains of action potentials. The red PSTH in $D$ shows grouped responses of three neurons to whisker deflection in a normal rat, and the black PSTH shows grouped responses of three other cells recorded in the same rat $3 \mathrm{~d}$ after kainic acid lesion of Zl. One of the latter cells was juxtacellularly stained with Neurobiotin (photomicrograph in $\boldsymbol{D}$ ). $\boldsymbol{E}$, Population PSTH of whisker-evoked responses in ZI-lesioned rats. Histogram in $\boldsymbol{F}$ shows the distribution of response latencies in that same population of neurons. Photomicrographs in $\boldsymbol{G}$ and $\boldsymbol{H}$ show NeuN immunostaining of Zl in the intact and lesioned side of the brain, respectively. Scale bars: D, $100 \mu \mathrm{m}$; H, $200 \mu \mathrm{m}$

hyperpolarizing events that reversed in sign during membrane hyperpolarization (Fig. 3B). These observations are thus consistent with the notion that the low excitability of Po neurons could be ascribed to a sustained barrage of IPSPs and that ZI likely contributes to this activity.

After an excitotoxic lesion of ZI, in most Po cells, the sustained barrage of IPSPs disappeared, the neurons exhibited rhythmic cell discharges (Fig. 3C), and responses to sensory stimulation 


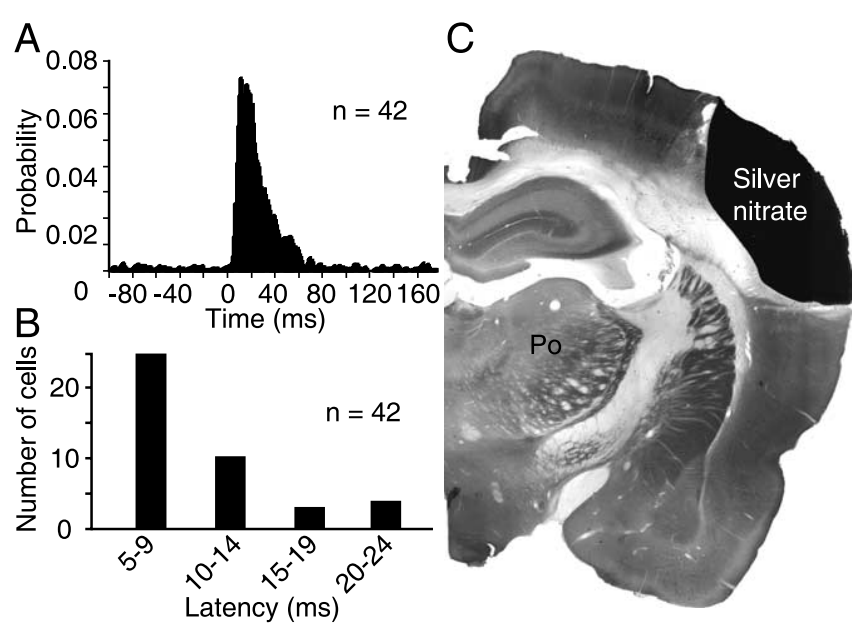

Figure 4. Whisker-evoked responses persist after cortical lesion in Zl-lesioned rats. A, Population PSTH of whisker-evoked responses after cortical lesion in Zl-lesioned rats. Histogram in $\boldsymbol{B}$ shows the distribution of response latencies in that same population of neurons. Photomicrograph in C shows the extent of the cortical lesion produced by an application of silver nitrate on the pia.

were markedly enhanced. The red PSTH in Figure $3 D$ shows responses of three Po cells that were recorded extracellularly in a rat before ZI lesion. It contains a small peak at $\sim 20 \mathrm{~ms}$ that barely reaches significance level ( $p=0.05$; one-tailed $t$ test). Recordings in the same rat $3 \mathrm{~d}$ after ZI lesion revealed Po neurons with increased spontaneous firing rates and prominent, short-latency responses to whisker deflection (Fig. 3D, black PSTH). Similar responses were observed in 46 additional cells in seven ZIlesioned rats (Fig. 3E, population PSTH). The mean latency of response onsets in that population was $11.68 \pm 5.96 \mathrm{~ms}$ (median, $11 \mathrm{~ms}$ ), with $46 \%$ of the cells responding at latencies $\leq 10 \mathrm{~ms}$ (Fig. $3 F)$. As confirmed by Nissl staining in all rats and NeuN immunostaining in three rats (Fig. $3 G, H$ ), ZI lesions involved a territory $\sim 800 \mu \mathrm{m}$ in diameter (mediolateral and anteroposterior), which included ZId, ZIv, and part of the subthalamic nucleus. Little, if any, cell loss was noticed in the reticular thalamic nucleus and in the anterior pretectal nucleus (APT).

\section{Sensory responses persist in ZI-lesioned rats after cortical lesion}

It was reported previously that whisker-evoked responses in Po are abolished during barrel cortex inactivation (Diamond et al., 1992b). We thus examined whether cortical lesion still suppresses responses in ZI-lesioned rats. The main effect of an extensive lesion of the barrel field was to abolish spontaneous rhythmic discharges, but cells maintained strong responses to whisker stimulation (Fig. 4A). Among a population of 42 cells recorded after ZI and cortical lesions ( $n=7$ rats), the mean latency of responses was $9.63 \pm 4.84 \mathrm{~ms}$ (median, $8 \mathrm{~ms}$ ) (Fig. $4 B$ ), which is statistically different from that computed after ZI lesion alone (i.e., $11.68 \pm 5.96 \mathrm{~ms} ; p=0.04$; two-tailed $t$ test). However, this difference clearly relates to the number of long-latency responses after lesion of the ZI alone because, when latencies $>15 \mathrm{~ms}$ were removed from both distributions, the mean latencies of response onset became nearly identical $(8.88 \pm 3.26 \mathrm{~ms}$ after ZI lesion alone and $8.64 \pm 2.96 \mathrm{~ms}$ after $\mathrm{ZI}$ and cortex lesions; $p=0.74$; two-tailed $t$ test). Likewise, response probabilities within a $40 \mathrm{~ms}$ time window after stimulus onset did not significantly differ in both population PSTHs after subtraction of mean prestimulus firing probabilities ( $p=0.29$; paired $t$ test). As shown in Figure
$4 C$ and supplemental Figure S1 (available at www.jneurosci.org as supplemental material), silver nitrate lesions damaged a large expanse of the barrel field and extended into the deep cortical layers. In summary, whisker-evoked responses in Po survive cortical ablation when ZI is not functional, demonstrating an effective peripheral drive to this nucleus.

\section{Whisker deflection evokes short-latency, fast-rising EPSPs in Po after ZI lesion}

We next examined the synaptic responses produced in Po cells by whisker motion in ZI-lesioned rats. When barrel cortex was intact, intracellular recordings were dominated by the occurrence of spontaneous discharges superimposed on rhythmic bouts of fast EPSPs that obscured the shape and time course of the EPSPs evoked by whisker deflection (Fig. 5A). Cells did respond, however, as confirmed by the PSTHs built from intracellular spikes (Fig. 5A, inset). When rhythmic spontaneous activities were abolished by cortical lesion, whisker-evoked EPSPs showed up as fast-rising events (rise time, 10-90\%; peak amplitude, $445 \pm 155$ $\mu \mathrm{s} ; n=21$ cells) with amplitudes of $3-8 \mathrm{mV}$ at rest (Fig. $5 B, C$ ). They grew in size during inward current injection and could trigger a low-threshold spike crowned with a burst of action potentials as cells were further hyperpolarized. EPSPs exhibited little jitter, and, on average, the first EPSP occurred $8.05 \pm 2.50 \mathrm{~ms}$ after stimulus onset ( $n=21$ cells in 7 rats) (Fig. $5 E$, see histogram). Spikes departed from that EPSP, or more often from temporal summation of EPSPs, which delayed cell firing.

\section{Feedforward inhibition blocks whisker-evoked responses in Po}

Although the first detectable event elicited in most Po neurons was an EPSP, in six additional neurons recorded after incertal and cortical lesions, an early IPSP preceded EPSPs. The short latency of these IPSPs (5-6 ms) made it unlikely that they resulted from the firing of reticular thalamic neurons, which suggests they could be mediated by ZI neurons that were spared by kainic acid lesion or by other inhibitory inputs to Po (see Discussion). This brings up the important issue of whether feedforward inhibition occurs fast enough to prevent responses to sensory stimuli in unlesioned rats. This issue could hardly be addressed in rats maintained under light ketamine anesthesia because IPSPs produced by the sustained firing of ZI cells contributed to obscure the onset of whisker-evoked responses in Po. We thus examined this question in urethane-anesthetized rats after lesion of the barrel cortex. In those rats, no Po cell recorded extracellularly or intracellularly responded to whisker motion. Intracellular recordings ( $n=23$ cells in 8 rats) revealed that the first synaptic event was a prominent IPSP with a cluster of synaptic potentials in the trough of the hyperpolarization (Fig. 5D) (supplemental Fig. S4, available at www.jneurosci.org as supplemental material). IPSPs started at a mean latency of $5.74 \pm 1.21 \mathrm{~ms}$ (Fig. 5E), which is significantly shorter than the mean latency of the first EPSPs recorded in ZI-lesioned rats ( $p<0.0001$; paired $t$ test), confirming that inhibition precedes excitation in Po neurons after whisker stimulation.

\section{Cell location}

Two points in the above results deserve additional precision. First, it must be emphasized that the disinhibitory effect of ZI lesion did not manifest in all Po neurons; reasons for this are considered in Discussion. Second, because vibrissal representation in Po is located next to the VPM (Diamond et al., 1992a), much care was taken to ensure that cells disinhibited by ZI lesion 
were actually located in Po. Figure 6 documents the localization of 42 of these units that were juxtacellularly or intracellularly labeled with Neurobiotin in different series of experiments. Most were clustered in dorsal Po, within the very same region that receives interpolaris and incertal terminals (supplementary Fig. S5, available at www. jneurosci.org as supplemental material) (Williams et al., 1994; Barthó et al., 2002). Doubtful cases $(n=6)$ in which cell bodies straddled the VPM-Po border were not included in the present report.

\section{Anatomical basis of peripheral driving afferents in Po}

Previous anatomical studies have demonstrated the innervation of Po and ZI by the collaterals of interpolaris axons (references in legend of Fig. 1), but the ultrastructural basis of the feedforward circuit suggested by our electrophysiological data have never been documented. It still remains to be established whether SpVi terminals make synaptic contacts on the proximal dendrites of Po neurons, and whether SpVi terminals in ZI form synapses with GABAergic neurons. The ultrastructural features of the GABAergic incertal projection in Po have been described previously (Barthó et al., 2002).

Injections of $\mathrm{BDA}$ into the whiskersensitive region of $\mathrm{SpVi}(n=3)$ resulted in the labeling of large terminals in Po. The precise location of the terminals relative to the VPM-Po boundary was determined by parvalbumin and calbindin immunostainings. Parvalbumin labels PrV terminals, thus outlining VPM (Williams et al., 1994), whereas calbindin is present in many Po neurons but not in the VPM (Celio, 1990; Frassoni et al., 1991; Williams et al., 1994). Both staining methods confirmed that most BDA-labeled terminals were located in Po, not in the VPM. They were principally concentrated in a shell-like region next to the VPM border, in which whisker-responsive Po neurons were found in the electrophysiological experiments (Fig. 6) (supplemental Fig. S5, available at www.jneurosci.org as supplemental material). In addition, some SpVi terminals were observed in the more medial part of Po, in the angular and the paracentral nuclei, and in the ventral lateral part of the VPM. Double-fluorescent staining for BDA and vGlut2, a marker of subcortical glutamatergic terminals (Fremeau et al., 2001; Land et al., $2004)$, revealed that all BDA-labeled terminals $(n=27)$ were positive for vGlut2 (Fig. S5, available at www.jneurosci.org as supplemental material).

The ultrastructure of the BDA-labeled terminals in Po was seen to conform to the classic RL-type profile (Ralston et al., 1988 ), in that they contain round (R) vesicles and are of large (L) size (Fig. 7). Their shape was either globular (major/minor diameter, $1.9-4.4 \mu \mathrm{m} / 1-2.5 \mu \mathrm{m}$ ) or elongated (up to $10 \mu \mathrm{m}$ ). All examined terminals $(n=17)$ established multiple asymmetrical synapses on the thick dendrites (number of synapses, 54) or dendritic appendages (number of synapses, 27) of relay cells. Partial reconstructions of labeled SpVi terminals $(n=5)$ from 26 con- secutive ultrathin sections revealed that a single SpVi bouton always formed $>10$ synapses on its postsynaptic target (mean, 16.2; range, 10-21), demonstrating the presence of multiple release sites in all cases. The average minor diameter of dendrites postsynaptic to the labeled terminals was much larger $(1.74 \mu \mathrm{m}$; $n=16)$ than that of randomly selected dendrites in Po $(0.54 \mu \mathrm{m}$; $n=200$ ), indicating a preferential innervation of proximal dendrites. All of the terminals were characterized by the presence of multiple rows of puncta adhaerentia-like specializations, also known as filamentous contacts (Lieberman and Spacek, 1997). Together, these ultrastructural features indicate that SpVi terminals in Po are nearly identical to those formed by lemniscal "driver" afferents in the VPM.

Large excitatory terminals have been occasionally shown to contain GABA in the forebrain (Sandler and Smith, 1991). To absolutely rule out the possibility that SpVi terminals contain GABA, postembedding immunogold GABA staining was used to establish the transmitter content of the terminals. None of the BDA-labeled terminals examined $(n=17)$ displayed GABA immunoreactivity (Fig. $7 B$ ). In the same material, we also characterized the BDA-negative terminals that contact the proximal dendritic segments $(n=8)$ in the vicinity of SpVi boutons. Of a population of 32 terminals identified in serial ultrathin sections, $28(87.5 \%)$ were GABAergic, two were RL profiles negative for both GABA and BDA (6.25\%), one was GABA negative $(3.07 \%)$ and conformed to the RS type profile (Ralston et al., 1988), and 

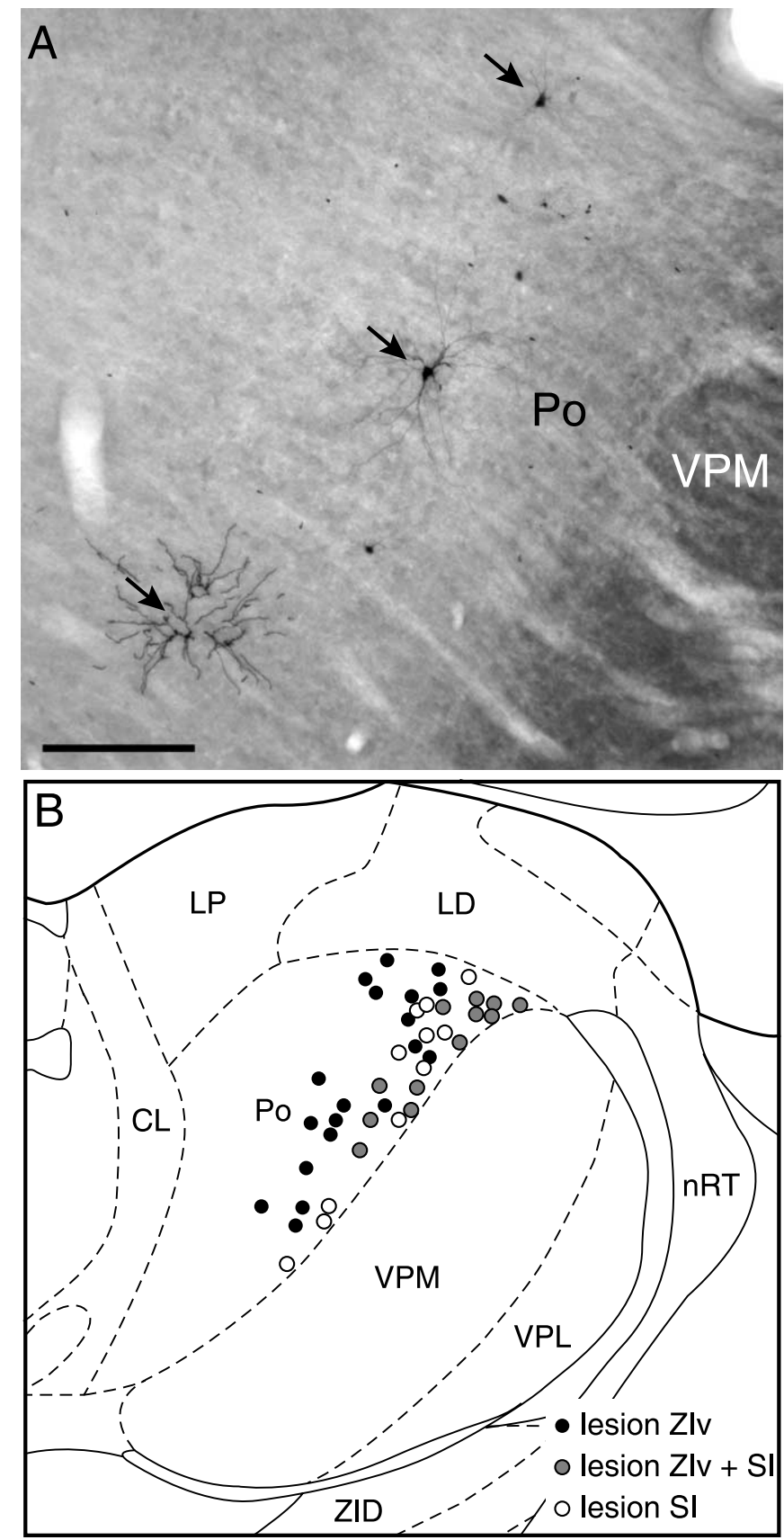

Figure 6. Location of $42 \mathrm{Po}$ cells that were juxtacellularly or intracellularly labeled in the course of electrophysiological experiments. Three labeled neurons are shown in $\boldsymbol{A}$ (arrows), and the drawing in $\boldsymbol{B}$ shows the location of each labeled unit on a representative frontal plane [ $\sim 3.30-3.80 \mathrm{~mm}$ behind bregma (Paxinos and Watson, 1998)]. Black dots, Cells recorded under ketamine anesthesia in Zl-lesioned rats; gray dots, cells recorded under ketamine anesthesia after Zl and cortex lesions; white dots, cells recorded under urethane anesthesia after cortical lesion. Scale bar, $200 \mu \mathrm{m}$. CL, Central lateral nucleus; LD, lateral dorsal nucleus; LP, lateral posterior nucleus; $\mathrm{nRT}$, reticular thalamic nucleus; $\mathrm{VPL}$, ventral posterior lateral nucleus.

one was unidentified. Previous studies have shown that Po receives GABAergic terminals from ZI and the APT, and that these terminals differ from those of reticular thalamic origin by their larger size, the presence of multiple release sites, and multiple filamentous contacts (Barthó et al., 2002; Bokor et al., 2005). On the basis of these criteria, even within the relatively short dendritic segments of $1.5 \mu \mathrm{m}$ examined, in three of eight cases, GABAergic terminals showing ZI/APT ultrastructure were found
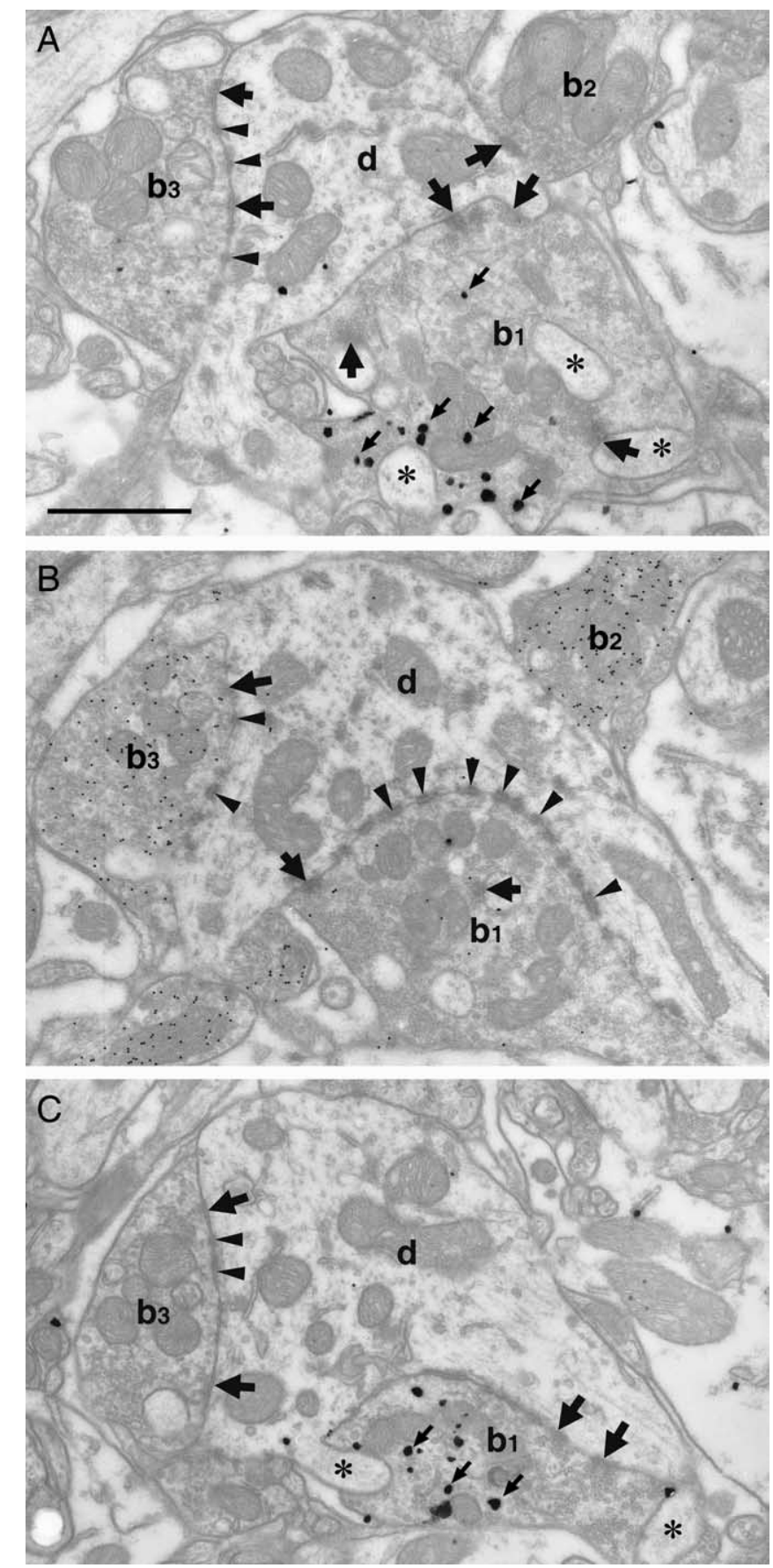

Figure 7. The ultrastructure of interpolaris terminals in $\mathrm{Po}$ is consistent with the classic features of peripheral afferents in the thalamus. $\mathbf{A}-\boldsymbol{C}$, High-power electron micrographs of a BDA-labeled SpVi bouton in three different, nonadjacent, ultrathin sections. Large black silver precipitates (small arrows) reveal the tracer, and small black dots in $\boldsymbol{B}$ show postembedding immunogold reactivity for $G A B A$. The silver signal for BDA is essentially missing in $B$ because of the etching procedure necessary for the postembedding reaction. The SpVi bouton (b1) is GABA negative and establishes asymmetrical synapses (arrows) on the thick dendrite (d) or the dendritic appendages (some of them labeled by asterisks). The terminal also forms several puncta adhaerentia-like contacts (arrowheads). The dendrite also receives symmetrical synapses from two GABAergic terminals (b2, b3). The smaller (b2) shows the ultrastructural features of an F terminal of reticular thalamic origin, whereas the larger (b3) forms multiple release sites and multiple rows of puncta adhaerentia-like contacts, which characterize GABAergic terminals of extrareticular origin. Scale bar, $1 \mu \mathrm{m}$.

to contact the same dendrites innervated by SpVi terminals (Fig. 7). These extrareticular terminals represented $10.71 \%$ of the GABAergic terminals $(n=28)$ on the proximal dendritic regions examined in our sample. 


\section{Anatomical basis of feedforward inhibition in ZIv}

Injections of BDA into the SpVi labeled a dense meshwork of terminals in ZIv, within the very same sector that contains cells retrogradely labeled or antidromically driven from Po. Preembedding double staining for BDA and GABA or parvalbumin (a marker of ZIv) (Covenas et al., 1991; Kolmac and Mitrofanis, 1998) demonstrated a precise overlap of the anterograde labeling with the territory of ZIv, with a number of BDA-labeled elements positioned around the somata of GABApositive (Fig. $8 A, B$ ) or parvalbuminpositive ZIv cells. Electron microscopic examination revealed that the labeled elements of SpVi origin are medium- to large-sized (1-5 $\mu \mathrm{m})$ terminals, which contain round vesicles and many mitochondria and form asymmetrical synapses with multiple release sites on the somata and proximal dendrites of GABAergic ZIv neurons. The somata of ZIv cells were also innervated by GABAergic terminals of unknown origin. Correlated light and electron microscopic examination revealed that the somata of individual ZIv neurons can receive contacts from multiple $\mathrm{SpVi}$ terminals, each containing several release sites. After complete reconstruction from serial ultrathin sections, a single SpVi terminal was found to establish 18 release sites on its GABA-immunoreactive postsynaptic target (Fig. $8 C-E$ ), which, to our knowledge, is one of the strongest innervation of a GABAergic neuron by an excitatory terminal in the entire forebrain.

\section{Discussion}

The main result of our study is that sensory transmission in Po is impeded by fast feedforward inhibition mediated by ZIv neurons. This result provides an explanation for the poor responsiveness of Po neurons to vibrissal inputs (Diamond et al., 1992a; Sosnik et al., 2001) and demonstrates that the difference in response magnitude in VPM and Po is not attributable to a difference in the strength of driving trigeminal afferents but to the presence of an efficient, selective GABAergic input in the latter nucleus. In addition, our data raises the important issue of whether the paralemniscal pathway is actually designed to relay messages produced by passive whisker deflection. Here we shall adopt a different viewpoint: namely, that sensory transmission in Po is controlled by a top-down disinhibitory mechanism, and that the paralemniscal pathway forwards to the cerebral cortex sensory information that is contingent on motor instructions.

\section{Methodological considerations}

In agreement with the observations of Trageser and Keller (2004), we found that not all Po neurons were disinhibited by ZI lesion. For the moment, it would be premature to conclude that nonresponsive cells represent a separate population of relay neurons, because failure of responses may be ascribed to methodological factors such as an incomplete lesion of ZI, non-optimal stimula- tion parameters, or because some recordings were made in more medial parts of Po that contain the forelimb and hindlimb representations (Diamond et al., 1992a). However, careful notation of all cells encountered in some individual descents that traversed the shell region of Po suggests that whisker-responsive and nonresponsive cells are intermingled. Thus, the possibility remains that, within that region, which receives the bulk of vibrissal inputs, separate populations exist (Trageser and Keller, 2004). Nonresponsive cells may represent a subpopulation of neurons that do not receive vibrissal input or again a distinct group of whisker-input recipient neurons in which vibrissal messages are gated by feedforward inhibition from the APT. Indeed, APT receives vibrissal input (Williams et al., 1994; Veinante and Deschênes, 1999; Veinante et al., 2000a), and a recent study has reported the presence of inhibitory pretectal afferents in Po, with ultrastructural features similar to those of ZIv afferents (Bokor et al., 2005).

\section{Anatomical basis of feedforward inhibition}

Results obtained in the present study are fully consonant with previous light microscopic observations pertaining to the anatomical organization of the paralemniscal pathway. They are in 
accord with the fact that ZIv receives a strong vibrissal input from PrV and SpVi (Nicolelis et al., 1992; Williams et al., 1994: Kolmac et al., 1998; Veinante and Deschênes, 1999; Veinante et al., 2000a), and that ZI projections to Po principally arise from ZIv (Power et al., 1999). Our ultrastructural data provide direct evidence that the collaterals of SpVi axons selectively terminate on the somata and proximal dendrites of GABAergic ZIv cells, indicating a potent peripheral drive, which is in line with the fast feedforward inhibitory action observed in Po.

Our data also confirm that SpVi input in Po principally distributes in a shell-like region over the VPM (Williams et al., 1994; Veinante et al., 2000a). Electron microscopic data unequivocally demonstrate that the poor responsiveness of Po neurons to whisker deflection is not attributable to a weak trigeminal input. In fact, SpVi terminals in Po are undistinguishable from the giant lemniscal terminals that mediate faithful relay of vibrissal messages in the VPM. The presence of large GABAergic terminals in the vicinity of the labeled SpVi boutons is also consistent with the fact that incertal axons terminate selectively on the proximal dendrites of Po neurons (Barthó et al., 2002). Although they represent a minority of GABAergic terminals in our sample, the strategic location of the giant terminals of extrareticular origin enables them to exert strong control over spontaneous and sensory-evoked activities in Po neurons.

\section{A matter of timing}

Two factors explain the virtual absence of whisker-evoked responses in our intact preparation: (1) the high rate of resting discharges of ZIv cells, which produces a sustained inhibitory shunt in Po neurons, and (2) feedforward inhibition. Nicolelis et al. (1992) already reported that, in rats maintained under light barbiturate anesthesia, ZIv neurons fire spontaneously at high rates. Whether ZIv cells do so in awake animals is not known, but this is not a crucial point for the present discussion, because sensory-evoked feedforward inhibition always occurs fast enough to block responses, even when ZIv cells discharge at low rate.

When ZIv cells do not discharge at high frequency, like in urethane anesthetized rats, feedforward inhibition prevails to decrease response probability and delay response onset. Two groups of inhibitory cells can take part in the feedforward inhibition: incertal and APT neurons, both of which receive vibrissal inputs from fast-conducting axons that arise from large multiwhisker cells in PrV and SpVi. The short latency of whiskerevoked responses in ZIv $(4.57 \pm 0.65 \mathrm{~ms})$ is clearly within the range expected from anatomical data and in agreement with latencies reported by Trageser and Keller (2004). Thus, after striking the vibrissae, ZI (and APT) cells will first be excited by collaterals of PrV axons that principally terminate in the caudal part of Po. After a short delay, say $\sim 2 \mathrm{~ms}$ to accommodate the longer path of the interpolaris circuit, the same cells will receive additional excitation from collaterals of interpolaris axons that project to the shell region of Po. Therefore, by the time SpVi input arrives in Po, ZI inhibition has already been activated by the PrV volley, and Po cells will fail to respond to the peripheral stimuli. Note that, regardless of the PrV impact on ZI, the arrival of SpVi input in Po is likely delayed with respect to ZI inhibition by a slowdown of conduction velocity in the axonal branches of parent SpVi fibers that divide at their entry in the thalamus (Veinante et al., 2000a). In summary, the connectivity of the system allows an unusual situation in which inhibition precedes ascending excitation, resulting in efficient shunting of the responses.
However, vibrissal messages also transit through VPM and activate layer 5 corticothalamic cells $\sim 12 \mathrm{~ms}$ after stimulus onset (Carvell and Simons, 1988; Armstrong-James et al., 1992). If one takes into account conduction time between cortex and thalamus (6-7 ms would be a reasonable figure given the small size of layer 5 axon collaterals that branch in Po) (Veinante et al., 2000b), corticothalamic EPSPs should occur in Po $\sim 18 \mathrm{~ms}$ after stimulus onset, which is actually close to the average response latencies reported previously by Diamond et al. (1992a) and Sosnik et al. (2001). Thus, this scenario provides an obvious explanation for the suppression of sensory responses after barrel cortex inactivation in urethane-anesthetized rats.

\section{Hypothesis of disinhibition}

Like VPM, Po receives vibrissal input from fast-conducting axons that contact large-diameter primary dendrites (Spacek and Lieberman, 1974; Varga et al., 2002; present study). In contrast to VPM cells, in which whisker deflection evokes EPSPs followed by IPSPs mediated by reticular thalamic cells, Po neurons are at once inhibited in a feedforward manner by ZI afferents. The fact that inhibition comes first suggests that the paralemniscal pathway is actually not designed to relay messages produced by passive whisker deflection but that the relay of vibrissal information might rely on a mechanism of disinhibition. This raises the questions of how and under which conditions disinhibition comes into action.

To create a window of disinhibition in Po, transient inhibition of ZIv cells should be time locked to the arrival of ascending inputs. Inhibitory cells that could suppress discharges in ZIv remain as yet unidentified, but there is an evidence that ZIv contains a rich network of collaterals that arise from ZId and ZIv neurons (Power and Mitrofanis, 1999; Barthó et al., 2002). Therefore, GABAergic neurons located within ZI itself might be involved. Because ZI receives dense excitatory inputs from layer 5 neurons of different cortical areas (Mitrofanis and Mikuletic, 1999), we propose that disinhibition operates in a top-down manner. In this way, sensory transmission in Po would depend on motor-related instructions that are sent to the brainstem by corticofugal axons. A top-down, motor-related, disinhibitory mechanism has already been demonstrated in the other major target of the ZIv, the superior colliculus. In awake monkeys, incertal neurons stop firing before saccade execution (Ma, 1996), and the duration of perisaccadic pauses positively correlates with the duration of saccades. Because the incerto-collicular pathway is also GABAergic (Ficalora and Mize, 1989; Kim et al., 1992; Nicolelis et al., 1992), this result strongly supports our proposal that the silencing of ZIv cells, and therefore disinhibition of their targets, operates in the context of motor activities. That Po is involved in processing sensory inputs in relation to whisking is further indicated by metabolic studies in which it was shown that whisker motion induced by motor cortex stimulation selectively increases deoxyglucose uptake in the interpolaris nucleus and dorsal Po, whereas passive whisker deflection increases glucose utilization principally in PrV and VPM (Sharp and Evans, 1982; Gonzalez and Sharp, 1985; Jacquin et al., 1993).

\section{References}

Armstrong-James M, Fox K, Das-Gupta A (1992) Flow of excitation within rat barrel cortex on striking a single vibrissa. J Neurophysiol 68:1345-1358.

Baimbridge KG, Miller JJ (1982) Immunohistochemical localization of calcium-binding protein in the cerebellum, hippocampal formation and olfactory bulb of the rat. Brain Res 245:223-229. 
Barthó P, Freund TF, Acsády L (2002) Selective GABAergic innervation of thalamic nuclei from zona incerta. Eur J Neurosci 16:999-1014.

Bender DB (1983) Visual activation of neurons in the primate pulvinar depends on cortex but not colliculus. Brain Res 279:258-261.

Bokor H, Frère SGA, Eyre MD, Slézia A, Ulbert I, Lüthi A, Acsády L (2005) Selective GABAergic control of higher order thalamic relays. Neuron 45:929-940.

Bourassa J, Pinault D, Deschênes M (1995) Corticothalamic projections from the cortical barrel field to the somatosensory thalamus in rats: a single-fiber study using biocytin as an anterograde tracer. Eur J Neurosci 7:19-30.

Carvell GE, Simons DJ (1988) Membrane potential changes in rat Sml cortical neurons evoked by controlled stimulation of mystacial vibrissae. Brain Res 448:186-191.

Celio MR (1990) Calbindin D-28k and parvalbumin in the rat nervous system. Neuroscience 35:375-475.

Chiaia NL, Rhoades RW, Fish SE, Killackey HP (1991) Thalamic processing of vibrissal information in the rat. II. Morphological and functional properties of medial ventral posterior nucleus and posterior nucleus neurons. J Comp Neurol 314:217-236.

Covenas R, De Leon M, Alonso JR, Arevalo R, Lara J, Aijon J (1991) Distribution of parvalbumin-immunoreactivity in the rat thalamus using a monoclonal antibody. Arch Ital Biol 129:199-210.

Diamond ME (1995) Somatosensory thalamus of the rat. In: Cerebral cortex, Vol 11 (Jones EG, Diamond IT, eds), pp 189-219. New York: Plenum.

Diamond ME, Armstrong-James M, Ebner FF (1992a) Somatic sensory responses in the rostral sector of the posterior group (POm) and in the ventral posterior medial nucleus (VPM) of the rat thalamus. J Comp Neurol 318:462-476.

Diamond ME, Armstrong-James M, Budway MJ, Ebner FF (1992b) Somatic sensory responses in the rostral sector of the posterior group (POm) and in the ventral posterior medial nucleus (VPM) of the rat thalamus: dependence on the barrel field cortex. J Comp Neurol 319:66-84.

Ficalora AS, Mize RR (1989) The neurons of the substantia nigra and zona incerta which project to the cat superior colliculus are GABA immunoreactive: a double-label study using GABA immunocytochemistry and lectin retrograde transport. Neuroscience 29:567-581.

Frassoni C, Bentivoglio M, Spreafico R, Sanchez MP, Puelles L, Fairen A (1991) Postnatal development of calbindin and parvalbumin immunoreactivity in the thalamus of the rat. Brain Res Dev 58:243-249.

Fremeau Jr RT, Troyer MD, Pahner I, Nygaard GO, Tran CH, Reimer RJ, Bellocchio EE, Fortin D, Storm-Mathisen J, Edwards RH (2001) The expression of vesicular glutamate transporters defines two classes of excitatory synapse. Neuron 31:247-260.

Gonzalez MF, Sharp FR (1985) Vibrissae tactile stimulation: (14C) 2-deoxyglucose uptake in rat brainstem, thalamus, and cortex. J Comp Neurol 231:457-472.

Hoogland PV, Welker E, Van der Loos H (1987) Organization of the projections from barrel cortex to thalamus in mice studied with Phaseolus vulgaris leucoagglutinin and HRP. Exp Brain Res 68:73-87.

Hoogland PV, Wouterlood FG, Welker E, Van der Loos H (1991) Ultrastructure of giant and small thalamic terminals of cortical origin: a study of the projections from barrel cortex in mice using Phaseolus vulgaris leuco-agglutinin (PHA-L). Exp Brain Res 87:159-172.

Jacquin MF, Barcia M, Rhoades RW (1989) Structure-function relationships in rat brainstem subnucleus interpolaris. IV. Projection neurons. J Comp Neurol 282:45-62.

Jacquin MF, McCasland JS, Henderson TA, Rhoades RW, Woolsey TA (1993) 2-DG uptake patterns related to single vibrissae during exploratory behaviors in the hamster trigeminal system. J Comp Neurol 332:38-58.

Kim U, Gregory E, Hall WC (1992) Pathway from the zona incerta to the superior colliculus in the rat. J Comp Neurol 321:555-575.

Kolmac C, Mitrofanis J (1998) Distribution of various neurochemicals within the zona incerta: an immunocytochemical and histochemical study. Anat Embryol (Berl) 199:265-280.
Land PW, Kyonka E, Shamalla-Hannah L (2004) Vesicular glutamate transporters in the lateral geniculate nucleus: expression of VGLUT2 by retinal terminals. Brain Res 996:251-254.

Lieberman AR, Spacek J (1997) Filamentous contacts: the ultrastructure and three-dimensional organization of specialized non-synaptic interneuronal appositions in thalamic relay nuclei. Cell Tissue Res 288:43-57.

Lin CS, Nicolelis MA, Schneider JS, Chapin JK (1990) A major direct GABAergic pathway from zona incerta to neocortex. Science 248:1553-1556.

Ma TP (1996) Saccade-related omnivectoral pause neurons in the primate zona incerta. NeuroReport 7:2713-2716.

Mitrofanis J, Mikuletic L (1999) Organisation of the cortical projection to the zona incerta of the thalamus. J Comp Neurol 412:173-185.

Nicolelis MA, Chapin JK, Lin RC (1992) Somatotopic maps within the zona incerta relay parallel GABAergic somatosensory pathways to the neocortex, superior colliculus, and brainstem. Brain Res 577:134-141.

Paxinos G, Watson C (1998) The rat brain in stereotaxic coordinates, Ed 4. London: Academic.

Pinault D (1996) A novel single-cell staining procedure performed in vivo under electrophysiological control: morpho-functional features of juxtacellularly labeled thalamic cells and other central neurons with biocytin or Neurobiotin. J Neurosci Methods 65:113-136.

Power BD, Mitrofanis J (1999) Evidence for extensive inter-connections within the zona incerta in rats. Neurosci Lett 267:9-12.

Power BD, Kolmac CI, Mitrofanis J (1999) Evidence for a large projection from the zona incerta to the dorsal thalamus. J Comp Neurol 404:554-565.

Ralston III HJ, Ohara PT, Ralston DD, Chazal G (1988) The neuronal and synaptic organization of the cat and primate somatosensory thalamus. In: Somatosensory integration in the thalamus (Macchi G, Rustioni A, Spreafico R, eds), pp 127-141. Amsterdam: Elsevier.

Sandler R, Smith AD (1991) Coexistence of GABA and glutamate in mossy fiber terminals of the primate hippocampus: an ultrastructural study. J Comp Neurol 303:177-192.

Sharp FR, Evans K (1982) Regional (14C) 2-deoxyglucose uptake during vibrissae movements evoked by rat motor cortex stimulation. J Comp Neurol 208:255-287.

Somogyi P, Hodgson AJ, Chubb IW, Penke B, Erdei A (1985) Antisera to $\gamma$-aminobutyric acid. II. Immunocytochemical application to the central nervous system. J Histochem Cytochem 33:240-248.

Sosnik R, Haidarliu S, Ahissar E (2001) Temporal frequency of whisker movement. I. Representations in brain stem and thalamus. J Neurophysiol 86:339-353.

Spacek J, Lieberman AR (1974) Ultrastructure and three-dimensional organization of synaptic glomeruli in rat somatosensory thalamus. J Anat 117:487-516.

Szabat E, Soinila S, Happola O, Linnala A, Virtanen I (1992) A new monoclonal antibody against the GABA-protein conjugate shows immunoreactivity in sensory neurons of the rat. Neuroscience 47:409-420.

Trageser JC, Keller A (2004) Reducing the uncertainty: gating of peripheral inputs by zona incerta. J Neurosci 24:8911-8915.

Varga C, Sik A, Lavallée P, Deschênes M (2002) Dendroarchitecture of relay cells in thalamic barreloids: a substrate for cross-whisker modulation. J Neurosci 22:6186-6194.

Veinante P, Deschênes M (1999) Single- and multi-whisker channels in the ascending projections from the principal trigeminal nucleus in the rat. J Neurosci 19:5085-5095.

Veinante P, Jacquin MF, Deschênes M (2000a) Thalamic projections from the whisker-sensitive regions of the spinal trigeminal complex in the rat. J Comp Neurol 420:233-243.

Veinante P, Lavallée P, Deschênes M (2000b) Corticothalamic projections from layer 5 of the vibrissal barrel cortex in the rat. J Comp Neurol 424:197-204.

Williams MN, Zahm DS, Jacquin MF (1994) Differential foci and synaptic organization of the principal and spinal trigeminal projections to the thalamus in the rat. Eur J Neurosci 6:429-453. 